\title{
Digital generation of acoustic stimuli
}

\author{
JOHN D. LOVELL and EDWARD C. CARTERETTE \\ Perceptual Systems Laboratory, Department of Psychology \\ University of California, Los Angeles, California 90024
}

A technique for digital generation of diverse acoustic waveforms is described. The method is embodied as a complete computer program coded in Fortran. We discuss the operation, implementation, and use of the program and its ease of adaptation for use on any computer.

Digital techniques of producing acoustic waveforms provide the experimenter with a tool of great power and flexibility in producing stimuli. Recent experiments such as those of Carterette, Friedman, and Lovell (1969) and Ahumada and Lovell (1971) have used signals which were constructed so as to have precisely specified spectra by means of summing sinusoids. The program described here uses this technique for generating diverse waveforms.

The program, SOUND, is a short, single main program written in Fortran. It should be easily adapted for use with any laboratory computer. Although a program such as described by Mathews (1969) could be used to obtain the same output as that produced by SOUND, it is far more complicated to implement and use. The program as described here is intended to be understood as a technique that can be modified if necessary to suit a particular laboratory. Input and output statements and array sizes should be altered to fit the needs of the experimenter.

A slightly modified form of SOUND is used routinely and successfully in the Perceptual Systems Laboratory with on-line experiments run under control of a Hewlett-Packard HP2 216-B Disc-Operating-System having 16,384 words of core memory. The word size is 16 bits. Random access of samples from the disk is possible and is a very important consideration. Magnetic tape digital storage and analog recording of SOUND generations have been done with success. Of course, use of the generated samples directly from the computer after conversion and smoothing is superior to their use after recording on magnetic tape inasmuch as several additional sources of noise, such as variations in tape speed and tape noise, are avoided.

The SOUND program is complete except for the Fortran "Write" statements that transfer the samples from the generated output buffer to a mass-storage device. In the form written here, the program assumes a 16-bit computer word. The two's complement integers produced can be converted to the form required by an unsigned binary digital-to-analog converter by complementing the sign bit. Thus, the maximum and minimum values would be mapped into Octal $(177777)$ and Octal (000000), respectively, and zero into Octal (100000).

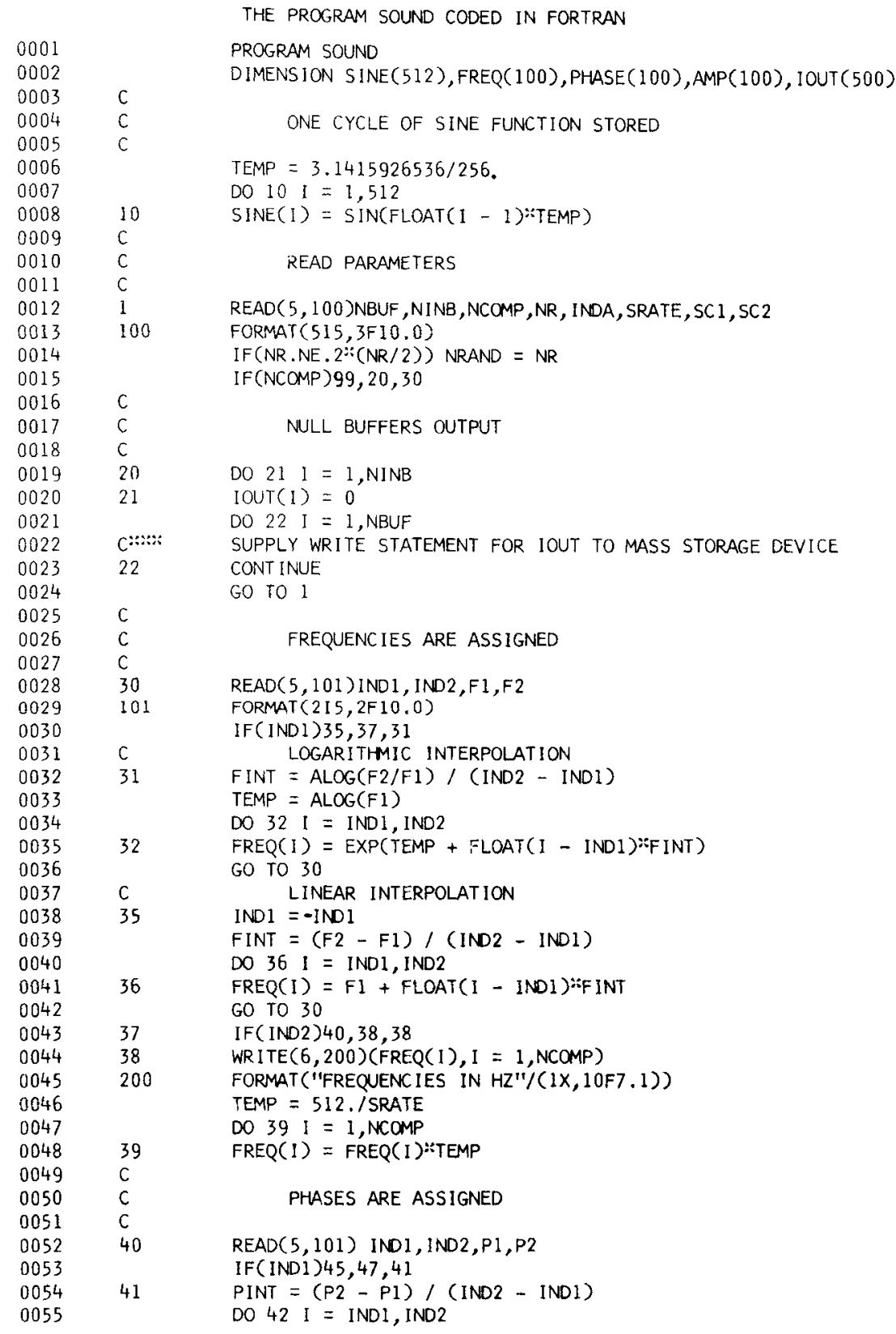

0001

0002

0003

0004

0005

0006

0007

0008

0009

0010

0011

0012

0013

0014

0015

0016

0017

0018

0019

0020

0021

0022

0023

0024

0025

0026

0027

0028

0029

0030

0031

0032

0033

0034

0035

0036

0037

0038

0039

0040

0041

0042

0043

0044

0045

0046

0047

0048

0049

0050

0051

0052

0053

0054

0055 


\begin{tabular}{|c|c|c|}
\hline $\begin{array}{l}0056 \\
0057\end{array}$ & 42 & $\begin{array}{l}\text { PHASE (I) }=P 1+\text { FLOAT( } 1-\text { INO1) } \because P \text { PINT } \\
\text { GO TO } 40\end{array}$ \\
\hline 0058 & 45 & DO $46 I=1, N C O M P$ \\
\hline 0059 & & NRAND $=$ NRAND $: 253$ \\
\hline 0060 & 46 & $\begin{array}{l}\operatorname{PHASE}(\mathrm{I})=\text { ABS(FLOAT(NRAND)) } / 64 . \\
\text { GO TO } 40\end{array}$ \\
\hline 0062 & 47 & IF (IND2) $50,48,48$ \\
\hline 0063 & 48 & $\operatorname{WRITE}(6,201)(\operatorname{PHASE}(I), I=1, \operatorname{NCOMP})$ \\
\hline 0064 & 201 & FORMAT ("PHASES O TO $\left.512^{\prime \prime} /(1 \times, 10 F 7.1)\right)$ \\
\hline 0065 & c & \\
\hline 0066 & c & AMPLITUDES ARE ASSIGNED \\
\hline 0067 & $\mathrm{c}$ & \\
\hline 0068 & 50 & $\operatorname{READ}(5,101)$ IND1, IND2,A1,A2 \\
\hline 0069 & & IF (IND1) $99,57,51$ \\
\hline 0070 & 51 & AINT $=(A 2-A 1) /(I N D 2-I N D 1)$ \\
\hline 0071 & & DO $521=$ INO1, IND2 \\
\hline 0072 & 52 & $\operatorname{AMP}(I)=A 1+F L O A T(I-I N D 1) \times A I N T$ \\
\hline 0073 & & GO TO 50 \\
\hline 0074 & 57 & If (INO2) $70,58,58$ \\
\hline 0075 & 58 & $\operatorname{WRITE}(6,202)(\operatorname{AMP}(1), 1=1, \operatorname{NCOMP})$ \\
\hline 0076 & 202 & FORMAT ("AMPLITUDES IN DB"/(1X,10F7.1)) \\
\hline 0077 & & SINPH $=0$ \\
\hline 0078 & & SPOWR $=0$ \\
\hline 0079 & & DO $591=1$, NCOMP \\
\hline 0080 & & $\operatorname{AMP}(I)=10 . \because(\operatorname{AMP}(1) / 20)$. \\
\hline 0081 & & $\operatorname{SINPH}=\operatorname{SINPH}+A M P(I)$ \\
\hline 0082 & 59 & SPOWR $=$ SPOWR + AMP $(1) \cdots 2$ \\
\hline 0083 & & TEMP $=20 . \because A L O G(S I N P H) / A L O G(10)$. \\
\hline 0084 & & SPOWR $=10$. ALOG(SPOWR $) / A L O G(10)$. \\
\hline 0085 & & WRITE $(6,203)$ TEMP, SPOWR \\
\hline 0086 & 203 & FORMAT ("SUM IN PHASE $=" F 7.2 " \mathrm{DB} " / "$ POWER SUM $=" \mathrm{~F} 7.2^{10 B "}$ ) \\
\hline 0087 & c & \\
\hline 0088 & c & AMPLITUDES ARE SCALED \\
\hline 0089 & c & \\
\hline 0090 & 60 & IF (INDA) $61,61,70$ \\
\hline 0091 & 61 & TEMP $=32767 . / 5 \mathrm{INPH}$ \\
\hline 0092 & & DO $62 \mathrm{I}=1, \mathrm{NCOMP}$ \\
\hline 0093 & 62 & $\operatorname{AMP}(1)=\operatorname{AMP}(1):$ TEMP \\
\hline 0094 & c & \\
\hline 0095 & c & GENERATE SOUND \\
\hline 0096 & c & \\
\hline 0097 & 70 & AINT $=(S C 2-S C 1) / F L O A T(N I N B \cdots N B U F-1)$ \\
\hline 0098 & & DO $79 \mathrm{~N}=1, \mathrm{NBUF}$ \\
\hline 0099 & & DO $78 \mathrm{I}=1, \mathrm{NINB}$ \\
\hline 0100 & & OUT $=0$. \\
\hline 0101 & & DO $77 \mathrm{~J}=1, \mathrm{NCOMP}$ \\
\hline 0102 & & $\operatorname{IF}(\operatorname{PHASE}(J), G E .511 .5) \operatorname{PHASE}(J)=\operatorname{PHASE}(J)-512$. \\
\hline 0103 & & OUT $=$ OUT + SINE(IFIXCPHASE $(J)+1.5) \because A M P(U)$ \\
\hline 0104 & & $\operatorname{PHASE}(\psi)=\operatorname{PHASE}(\mathrm{J})+\operatorname{FREQ}(j)$ \\
\hline 0105 & 77 & CONT INUE \\
\hline 0106 & 78 & IOUT $(I)=(S C 1+F L O A T((N-1) * N I N B+I-1) \because A I N T):$ OUT \\
\hline 0107 & co: & SUPPLY WRITE STATEMENT FOR IOUT TO MASS STORAGE DEVICE \\
\hline 0108 & 79 & CONTINUE \\
\hline 0109 & & GO TO 1 \\
\hline 0110 & 99 & END \\
\hline
\end{tabular}

\section{IMPLEMENTING THE PROGRAM}

Logical Units 5 and 6 are used as the standard input ( $5=$ tape reader) and output ( $6=$ teleprinter $)$ units. Lines 22 and 107 in the program listing should be replaced with output statements to write NINB values in the array IOUT on an appropriate output device. Initially, these values can be printed out in order to determine that the program is working properly. As described, the program will run if only the system control statements are added; but without replacing Lines 22 and 107 , the generated waveforms will not be output.

The dimensions of the FREQ, PHASE, and AMP arrays, and the IOUT array should be changed to fit individual needs. The maximum value of NCOMP is the dimension of the FREQ, PHASE, and AMP arrays. The the leftmost bit. Thus, the value of Bits 31-19 of a 32-bit computation are equivalent to the value of Bits 15-0 of a 16-bit computation. Also, the maximum unscaled amplitude is related to the bit size by the relation $(\mathrm{b}-1) * 6 \mathrm{~dB}$. For our machine $(16-1) * 6 \mathrm{~dB}=90 \mathrm{~dB}$. The amplitude of the final sound output also depends on the conversion process which will be briefly discussed below.

\section{OPERATION OF THE PROGRAM}

A brief synopsis of the way in which the program works will be useful. The steps are:

(1) A single cycle of a sine wave is generated.

(2) The user furnishes the values which specify: (a) the number of buffers to be generated (NBUF); (b) buffer size (NINB); (c) the number of sinusoidal components (NCOMP); (d) a random number (NR) for use in assigning phases (if NR is odd, it will be used as a starting number for the pseudorandom number generator; if NR is even, it produces no effect); (e) an indicator which determines whether or not the amplitudes of the components are to be multiplied by a gain factor such that the in-phase sum equals $90 \mathrm{~dB}$ (INDA); (f) the number of samples per second (NSAMP); (g) the initial and final values of a linear envelope (SC1 and SC2, respectively).

(3) A zero line (corresponding to a quiet interval) will be generated if the value of NCOMP is set to zero; and the program returns to Step 2 (see Example 1). Otherwise, frequencies, amplitudes, and phases of the components are specified (see Example 2).

(4) The index number of the first and last component is given (IND1 and IND2) and the corresponding frequencies of these components (F1 and F2). Components whose indices lie between IND1 and IND2 are assigned frequencies interpolated between F1 and F2. Interpolation can be linear (if IND1 is preceded by a minus sign) or logarithmic. (Note that this specification sequence can be repeated as often as required to assign all values and is terminated when IND1 is set equal to zero. Frequencies are assigned values in hertz, but are converted internally to increments per sample.)

(5) Phase assignments are made in the same fashion as those for frequency. The first and last values to be assigned (P1 and P2) must be in the range of 0 to 512 corresponding to 0 to $360 \mathrm{deg}$ or, equally, to 0 to $2 \pi$ radians. Phase values are not converted internally. If IND1 is set to -1 , phases are assigned randomly over the range of 0 to 512 . 


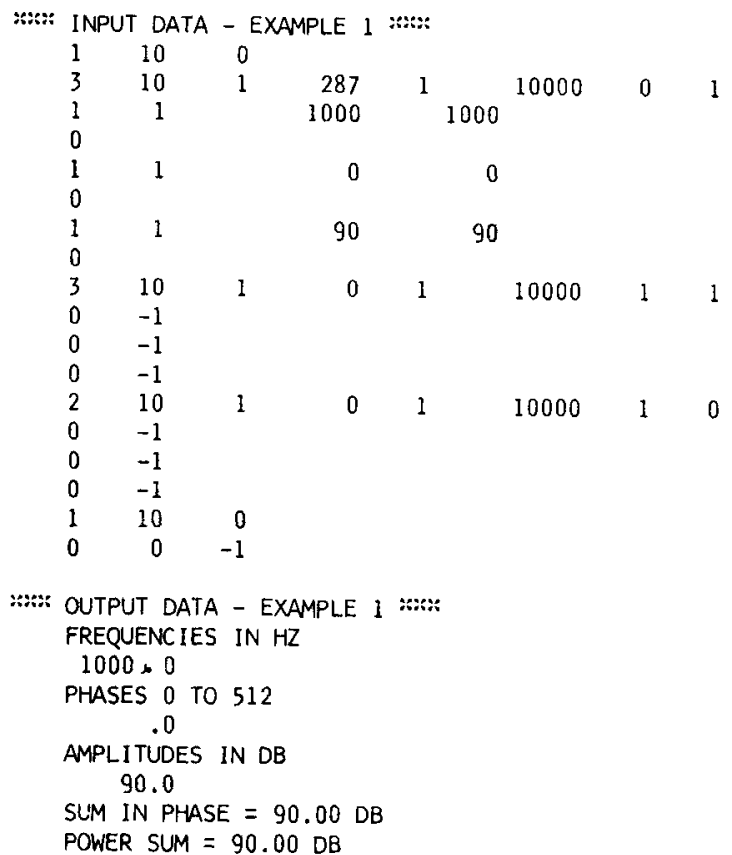

(6) Amplitudes are assigned as in 4 and 5 with the first and last values (A1 and $A 2$ ) in decibels. If INDA has been set to zero, amplitudes may be assigned relative to any base since they will be scaled to the maximum permissible in-phase sum. Otherwise, amplitudes should be assigned decibel values relative to the number 1 (see Example 2). Amplitudes are converted internally to a multiplier of a sine wave, which ranges between -1 and $+1$

(7) The specified waveform is generated. Upon completion, the program returns to Step 2 and the program is ready to continue.

\section{USING THE PROGRAM}

The program is capable of producing a close approximation to any steady-state waveform with superimposed linear changes in amplitude. Example 1 shows the computed output representing a $1,000-\mathrm{Hz}$ tone sampled at 10,000 samples per second. The tone is gated on in $3 \mathrm{msec}$ and gated of in 2 msec. The waveform produced in Example 1 is shown in Fig. 1a.

Example 2 shows the computed output of a complex waveform generated at 5,000 samples per second. The waveform is illustrated in Fig. 1b and its associated spectrum in Fig. 1c.

When constructing wideband noises, it is advisable to space components logarithmically to avoid beating in the complex. The result approximates pink noise if all component amplitudes are set equal or white noise if component amplitudes rise at a rate of $3 \mathrm{~dB}$ per octave. Phases can be either

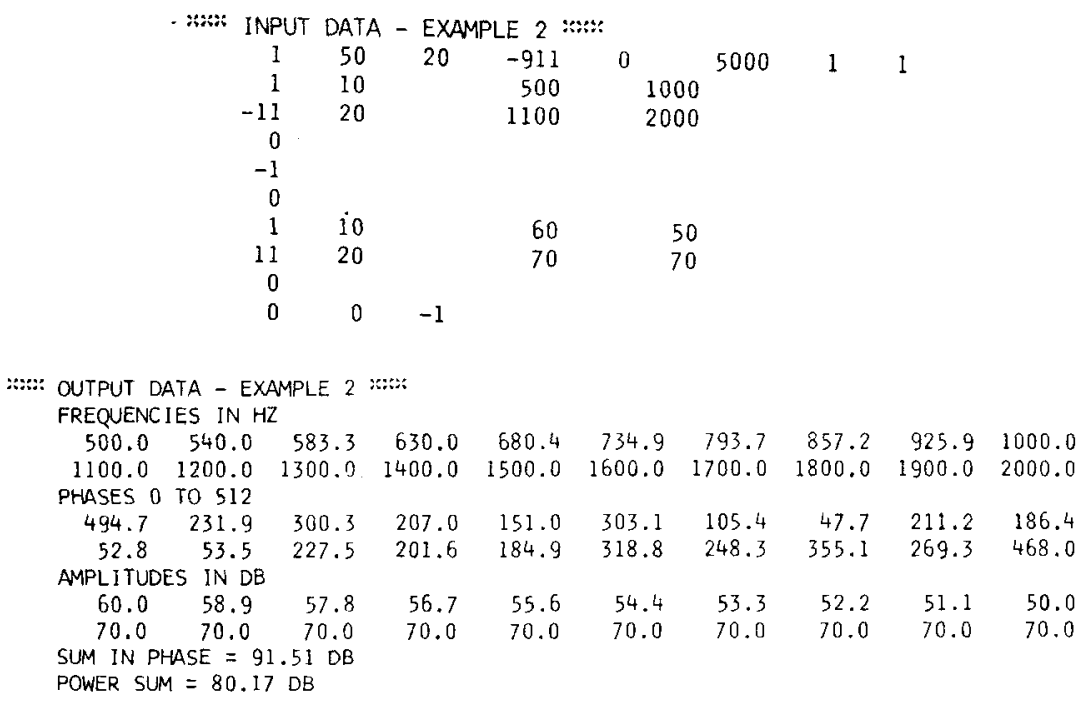

randomized or interpolated over the range 0 to 512 , corresponding to the range 0 to $2 \pi$.

An alternative to producing onsets and offsets with interpolated scale values is to use the phenomenon of beats to produce S-shaped amplitude functions having continuous first derivatives. For example, to turn on a $1,000-\mathrm{Hz}$ tone in $10 \mathrm{msec}$, a $975-\mathrm{Hz}$ tone with a phase angle of 0 and a $1,025-\mathrm{Hz}$ tone with a phase angle of $\pi$ are turned on for $10 \mathrm{msec}$. Then the frequencies of both tones are changed to $1,000 \mathrm{~Hz}$ without changing their phases (or a single $1,000-\mathrm{Hz}$ tone is continued with 6-dB greater amplitude.) Offset is produced in just the reverse manner.

In general, an arbitrary complex may be gated on and off by applying the same operation to each sinusoidal component of the complex. If $t$ is the rise or decay time in seconds, then each component frequency, $f$, is represented during $t$ by $f-(1 / 4 t)$ and $f+(1 / 4 t)$. The phases of each pair must differ by $\pi$ at the start of onset and at the end of offset.

\section{SOME SOURCES OF ERROR}

Increment in Sampling Stored

Function Not Always Integral

Different frequencies are realized from the stored function-one cycle of a sine wave-by varying the size of the step, that is, by varying the increment between samples taken at successive instants of sampling. If $I$ is the increment in samples, $L$ the function length, $f$ the frequency in hertz, and $S$ the sampling rate,

$$
I=f L / S
$$

Obviously, with L and S fixed, the increment must increase if frequency increases. If $f=5,000 \mathrm{~Hz}$ and $S=$ 10,000 , the increment I must be $256=$ $(5,000)(512) / 10,000$, and only two samples will be taken per cycle of the sampled waveform. If the frequency is halved to $2,500 \mathrm{~Hz}$, the increment is also halved, to 128 , but the number of samples taken per cycle doubles to four. In general, the increment, I, will not be an integer and when it is not, the sum of the increments will differ from the values of $L=1,2,3, \cdots$, 512. This difference is an error.

Mathews (1969) assessed the rms error in computing sine $\mathrm{X}$ by various methods and function lengths. It was found that "In general, rounding is about twice as accurate as truncation, and doubling the length of the stored function doubles the accuracy for both the truncation and rounding. Doubling the function length quadruples the accuracy for the interpolation method, however [p. 138]." 

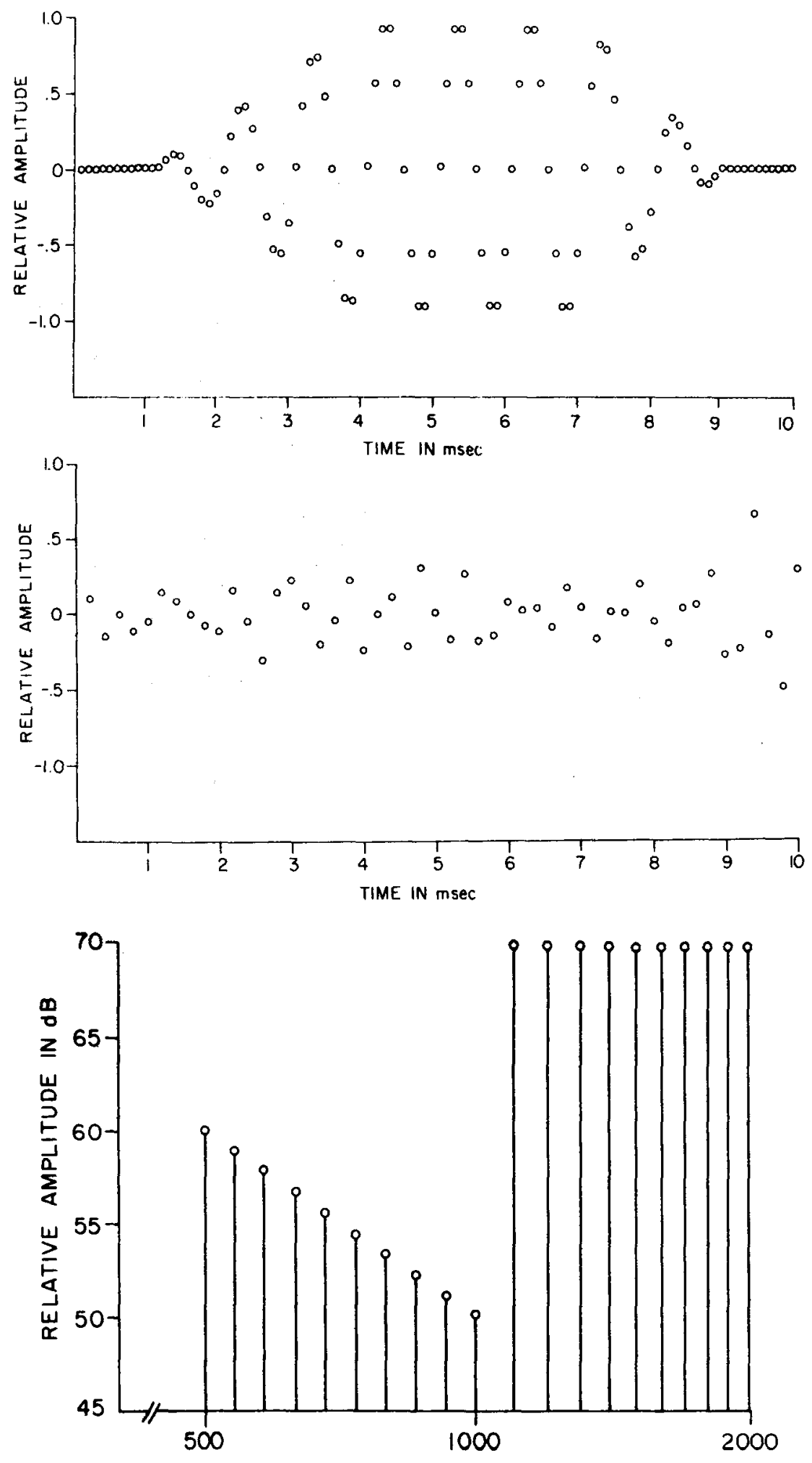

FREQUENCY IN Hz

Fig. 1. (a) (Example 1). A 1,000-Hz gated sinusoid. The rise time is $3 \mathrm{msec}$ and the decay time is 2 msec. Generation was at 10,000 samples per second. (b) (Example 2). A complex waveform generated at 5,000 samples per second. Phases of the components were assigned at random. (c) (Example 2). The amplitude-frequency spectrum of the waveform shown in (b).
In SOUND, the method of rounding has been used. Thus, the error in computing sine $\mathrm{X}$ is about $0.2 \%$, since the function length was 512 .

\section{Quantization Error}

Another source of error results from the quantization of the input data to the digital-to-analog converter. If the quantization is fine enough and if the quantized signal is well behaved relative to the sampling rate, then it can be shown (see Bendat \& Pierson, 1966, pp. 281-282) that the effect of quantization is like that of an additive random noise at the input. The mean square value of this noise is $q^{2} / 12$, where $q$ is the elementary quantum. When the full range of the signal is quantized at $2^{\mathrm{n}}$ steps, $q=1 / 2^{\mathrm{n}}$. The rms noise-to-signal ratio is thus $q / \sqrt{12}$ $\cong(.2889)$ q. As Table 1 shows, an 8-bit converter should suffice for many purposes, since the quantization noise is about $60 \mathrm{~dB}$ below the signal. A somewhat larger dynamic range is often desirable in the case of acoustic signals and it is prudent according to Mathews (1969) to use at least 12 bits, as we do in our laboratory. It is now easy to buy converters with 14- and 16-bit accuracy on the open market.

\section{Errors of Aliasing}

When a voltage function contains frequencies higher than $\mathrm{S} / 2 \mathrm{~Hz}$, where $S$ is the sampling rate, the resultant sound is heard at frequencies between 0 and $\mathrm{S} / 2 \mathrm{~Hz}$. This property, called aliasing or foldover, is an unavoidable consequence of sampling at a fixed time interval $t=1 / \mathrm{S}$ sec. Only those frequencies below the cutoff frequency

$$
\mathrm{f}_{\mathrm{c}}=\mathrm{S} / \mathbf{2}
$$

also known as the Nyquist frequency, are useful. In practical work with sound, these undesirable frequencies are removed by means of a low-pass smoothing filter placed in the circuit after the converter. The cutoff of the filter is set to a frequency below $f_{c}$ so that frequencies at or above the Nyquist frequency are about $60 \mathrm{~dB}$ below the level of the filter-cutoff frequency. Bendat and Piersol (1966) state that it is a good rule to select the Nyquist frequency to be 1.5 to 2 times the maximum frequency of interest. This is certainly a good rule, though a bit conservative. In our laboratory, we typically sample at 20 or $10 \mathrm{kHz}$ and set the lowpass filter cutoffs at 8 or $4 \mathrm{kHz}$, respectively. We use active filters. Mathews (1969) describes the 
Table 1

RMS Quantization Noise

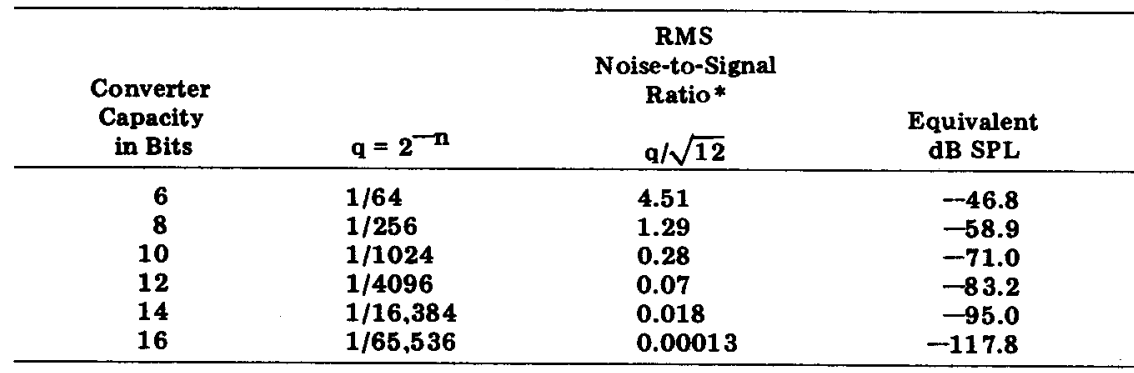

*Times $10^{-3}$

construction of excellent passive smoothing filters.

\section{DISCUSSION}

The method discussed above is simple and relatively straightforward. It may not be obvious that the technique is extremely versatile. Noise bands can be generated with precise control over the amplitude, frequency, and phase relationships. Spectra are easily generated which have the same Fourier component amplitudes as those of square or triangular waves, of sampling and computational noise may be found in Kaiser's (1966) article and Gold and Rader's (1969) book. A thorough theoretical and practical treatment of spectral analysis and their statistics is given by Jenkins and Watts (1968), as well as by Bendat and Piersol (1966).

\section{REFERENCES}

AHUMADA, A., JR., \& LOVELL, J. D. Stimulus features in signal detection. Journal of the Acoustical Society of America, 1971, 46, 1751-1756.

BENDAT, J. S., \& PIERSOL, A. G. Measurement and analysis of random data. New York: Wiley, 1966.

CARTERETTE, E, C., FRIEDMAN, M. P., \& LOVELL, J. D. M ach bands in hearing. Journal of the Acoustical Society of America, 1969, 45, 986-998.

GOLD, B., \& RADER, C. Digital processing of signals. New Y ork: M cGraw-Hill, 1969. JENKINS, G. W., \& WATTS, D. G. Spectral analysis and its applications. San Francisco: Holden-Day, 1968.

KAISER, J. F. Digital filters. In F. F. Kuo and J. F. Kaiser (Eds.), System analysis by digital computer. New York: Wiley, 1966.

MATHEWS, M. V. The technology of computer music. Cambridge: M.I.T. Press, 1969. 